\title{
La solitude du discours amoureux aujourd'hui. Réflexions à partir de Roland Barthes et une critique*
}

\author{
Chiara Piazzesi
}

On écrit avec son désir, et j'en finis pas de désirer. (Roland Barthes)

\section{La solitude du discours amoureux}

En 1977, tâchant de motiver aux yeux du lecteur l'aventure littéraire qu'il était en train de lui offrir, Roland Barthes ouvrait ses Fragments d'un discours amoureux en observant que le discours amoureux se trouvait, à l'époque, dans une condition d'extrême solitude, abandonné par les langages environnants, « ou ignoré, ou déprécié, ou moqué par eux, coupé non seulement du pouvoir, mais aussi de ses mécanismes (sciences, savoirs, arts) (Fragments 27). Qu'est-ce que cela signifie? Et pourquoi répondre, comme Barthes le fait, par une «performance » du discours amoureux, telle que le sont les Fragments? Je voudrais proposer une lecture de cette affirmation énigmatique et fascinante concernant la solitude du discours amoureux - et, par ce biais, quelques réflexions sur le discours amoureux en tant que tel; ensuite, discuter de manière critique une tentative récente de réponse à l'appel barthésien, pour en tirer quelques considérations supplémentaires sur l'écoute que la philosophie pourrait offrir au discours amoureux en tant que cadre fondamental d'expérience.

Au premier regard, les mots de Barthes pourraient superficiellement être lus comme une légitimation toujours disponible de tout discours ou réflexion sur l'amour, comme si la 
dénonciation de Barthes signifiait qu'on ne parlait pas assez de l'amour et des relations amoureuses à l'époque (ce qui n'est pas très probable). En d'autres termes, on risque de prendre l'affirmation de Barthes comme si elle sollicitait une intensification du «méta-discours » amoureux (c'est-à-dire du discours sur l'amour). Bien au contraire, lorsqu'il explique la construction de son livre, Barthes souligne de manière très claire la distinction entre discours et méta-discours amoureux. La structure même des Fragments répond à la tentative d'éviter autant que possible le méta-discours, pour affirmer le discours amoureux. Cela est un indicateur pour comprendre l'expression «discours amoureux » ainsi que la remarque sur son extrême solitude. On sait que derrière les Fragments d'un discours amoureux se trouve un long travail d'analyse «technique » et structurelle de Barthes sur le discours amoureux et sur sa tradition ${ }^{1}$. Ce travail est pourtant presque ôté dans les Fragments qui se déploient, contrairement aux notes de cours des années 1974-1976, en tant que performance du discours amoureux — c'est-à-dire en discours amoureux.

À la lumière de ces considérations, nous allons tout d'abord nous demander qu'est-ce que le discours amoureux que Barthes considère comme étant abandonné. On commencera par un aperçu sur les caractéristiques du discours amoureux, pour montrer en quelle mesure son incohérence et sa fragmentation lui sont constitutives. Ensuite, on analysera le projet barthésien de restituer une dignité à ce discours et à l'expérience qu'il véhicule — de le soustraire à sa solitude. Pour conclure, on montrera pourquoi certaines attaques moralisatrices contemporaines envers l'instabilité de l'expérience amoureuse actuelle, au lieu d'offrir un espace d'écoute au discours amoureux, finissent souvent par le réduire au silence : insoucieuses d'une confrontation profonde avec le discours amoureux courant, ces attaques ne créent pas les conditions pour le 
dépassement des tensions constitutives de la sémantique amoureuse occidentale dans la modernité, mais risquent d'en répliquer le schéma et d'accentuer les malaises qui en dérivent.

\section{À propos de la linguistique du discours (amoureux)}

Grammar is a piano I play by ear. All I know about grammar is its power. (Joan Didion)

Certains outils théoriques du Barthes linguiste et sémiologue peuvent aider à comprendre la nature et le statut du discours amoureux. Dans un essai sur «La linguistique du discours » (1970) — définie comme translinguistique —, Barthes fournit des précisions importantes à propos de l'objet de cette discipline, idéalement unificatrice de l'ensemble des approches linguistiques, tout en essayant de la distinguer de la linguistique «simple ». Celle-ci s'occupe de la phrase et ne peut pas (ou bien refuse de) dépasser ce niveau; celle-là en revanche prend en charge l'analyse du discours, se propose d'aller au-delà du sens de la phrase pour la reconduire à sa «référence ». La linguistique du discours s'engage dans une opération systématique très proche de celle qu'on définit par contextualisation. Dans la rhétorique, par exemple, ancêtre de la translinguistique, la distinction entre les situations de discours (mimétique, délibérative, judiciaire, épidictique, etc.), qui sert à codifier les mots, se fait par la référence extra-linguistique et intra-sémiologique. La linguistique du discours s'occupe de cette dimension de la référence. De plus, elle travaille selon un principe d'intégration, à la recherche de l'articulation du système linguistique sur la praxis historique et sociale.

Un savoir translinguistique soucieux d'analyser le discours amoureux s'occuperait donc de retracer non pas son «sens », mais sa valence ou sa fonction de praxis. D'après Barthes, on vient de le voir, l'analyse de la signification des mots employés ou du sens des phrases formulées ne 
suffit pas à épuiser l'étude linguistique des énonciations et des schémas d'énonciations par rapport à un « discours ». Il y a encore la dimension de praxis, impliquant un héritage historique, une construction culturelle, capturés et réactualisés dans les structures et les règles plus ou moins codifiées du jeu social, maîtrisés en cette forme par les acteurs qui s'expriment selon ses règles. Il s'agit en gros de l'aspect du langage et de l'action que Wittgenstein aurait définit «grammatical», rassemblant l'ensemble des usages possibles (que la définition de la signification du mot n'épuise jamais) d'un mot ou d'une proposition à l'intérieur d'un contexte social ou culturel. Pour essayer de décrire ce surplus il faut inclure dans la description les implications de l'idée de référence introduite par Barthes : ce n'est pas le contexte qui fait le sens d'une proposition, mais c'est plutôt la connexion compétente entre contexte et proposition qui fait à la fois le sens de la proposition et qui définit le contexte en tant que tel. Les Fragments mêmes nous donnent des indications sur la portée spécifique de la dimension sociale de cette pratique linguistique de contextualisation et signification. En lui présentant la structure de son ouvrage, Barthes attire l'attention du lecteur sur certaines caractéristiques de celui-ci, qui nous donnent une idée plus précise de cette dimension de la pratique de discours.

En premier lieu, on l'a vu, Barthes souligne la différence entre discours et méta-discours : son ouvrage se tiendra exclusivement au premier. Ensuite, il explique le statut et la fonction des figures du discours amoureux (unités thématiques de la sémantique amoureuse traditionnelle, telles que, par exemple, «Adorable », «Attente », «Catastrophe », « Drame », « Déclaration », « Dédicace », « Magie », « Nuit », «Pleurer », « Pourquoi », «Seul», « Union », etc.), qui constituent les étapes de l'ouvrage et de la performance du discours amoureux : comme le schema est, au sens originaire, «le geste du corps saisi en action », ainsi chaque figure dans les Fragments saisit «l'amoureux au travail», en train de pratiquer et déployer le discours 
amoureux. Mais il y a plus : les figures, en ce sens, «se découpent selon qu'on peut reconnaître, dans le discours qui passe, quelque chose qui a été lu, entendu, éprouvé ». Elles ont par conséquent à la fois le statut d'un signe et celui d'une image ou d'un récit : elles sont fondées dans la capacité de la personne (du lecteur) de reconnaître la « scène de langage » (qui n'est pas simplement une phrase, mais une phrase contextualisée, par rapport à laquelle on peut introduire des variations en faisant abstraction du contexte) correspondante et d'y articuler sa propre expérience, sa propre histoire. En outre, Barthes ajoute que pour former les figures «il ne faut ni plus ni moins que ce guide: le sentiment amoureux »(Fragments 30). Schéma partagé de mouvement coordonné, composante d'une chorégraphie codifiée mais en même temps toujours créative, acte expressif novateur et pourtant reconnaissable en tant que partie du code d'une praxis qu'il contextualise, geste individualisé dont la forme gestuelle est signifiante puisque partagée avec les autres acteurs, la figure du discours (amoureux) est alors une forme d'expression d'une sorte de lieu commun du sentiment, de l'attitude émotive du sujet (amoureux) par rapport à sa situation. On pourrait dire aussi : la figure est le frame de transmission de la situation émotive du sujet qui parle et qui en même temps formule cette situation par le biais de la figure. Pourtant, la figure n'est pas un mécanisme mort, vivifié par le sentiment de celui qui l'emploie : il y a, au contraire, par rapport au potentiel expressif de la figure de discours, une analogie de fonctionnement entre celle-ci et ce que Marcel Mauss définit comme techniques $d u$ corps — rapprochement qui m'a été suggéré par la référence de Barthes à la figure comme geste du corps (amoureux).

Mauss compare dans son travail de 1936 une série de techniques sociales ayant pour but l'éducation des corps à certaines activités sociales spécifiques (la nage, la marche, la posture à table, la course, la posture des mains en repos, etc.). Ses observations concernent l'examen des 
régularités et des particularités culturelles de certaines pratiques de posture corporelle, mais surtout l'approfondissement de la dimension à la fois somatique, émotive, affective et rationnelle qu'elles possèdent en tant que performances vives d'une appartenance sociale. Les techniques du corps sont, en ce sens, une modalité d'expression qui définit l'espace, le rythme, l'orientation symbolique d'un contexte par l'articulation de la présence corporelle et de son intentionnalité grâce à un système de significations et attributions de valeur incorporées. D'autre part, elles articulent l'émotivité individuelle liée à l'expression somatique envisagée ou adoptée, en fonction de dispositions apprises qui façonnent non seulement les muscles et le corps, mais les affects et l'évaluation affective des contextes mêmes, c'est-à-dire la manière de «se sentir » en performant un geste plus ou moins chargé de signification symbolique. À chaque occurrence, le schéma gestuel est réactualisé par la situation (spatiale, émotive, perceptive, projective, symbolique) personnelle, donc par l'histoire personnelle, qui le rend action vivante, geste personnel expressif. En ce sens, Mauss se sert du terme habitus ${ }^{2}$, qui sera une notion majeure dans le cadre de la théorie sociologique de Pierre Bourdieu, pour indiquer l'effet de matrice de l'apprentissage individuel des techniques du corps : elles participent d'un savoir dispositionnel spécifiquement social, articulent une cosmologie de significations subjectives/objectives, dans laquelle l'émotivité liée à la posture fait référence au contexte contingent, mais en même temps lui imprime sa morphologie axiologique. Les techniques du corps façonnent ainsi une psychologie entière incarnée ${ }^{3}$.

Ce qui nous intéresse ici est la correspondance interne entre éducation de la gestualité, accommodement somatique et intensité émotive. Le geste exprime la réaction émotive et en même temps la technique pour maîtriser cette émotivité, pour lui donner une forme : le geste calme et contrôlé du sang froid se produit avec l'émotion correspondante, il est l'effort 
somatique d'accommodement qui intensifie une certaine nuance affective, lui donne dignité expressive, se situe dans le contexte et situe le contexte même (par l'attribution de significations, la définition de rapport spatiaux codifiés, la limitation des légitimités). Or, il me semble que la figure chez Barthes ait une portée et une fonction extrêmement proches de celles de la technique du corps. Geste linguistique codifié, appartenant à un ordre de discours ayant ses règles et par conséquent ses espaces d'improvisation et de réactualisation individuelle; occasion d'identification et de reconnaissance, et ainsi de continuation du discours; instrument performé de «communication », qui crée une communauté d'expérience et d'expression, la figure — qui n'est jamais isolée, mais qui s'affirme en tant que partie unitaire d'une multiplicité organisée correspond à une disposition à la fois linguistique et émotive. Actualisée dans l'affirmation par l'épisode émotif réactivant la disposition, la figure actualise à son tour l'émotion correspondante : elle fonctionne comme un intensificateur de l'émotion et crée le cadre pour son expression articulée ${ }^{4}$. La figure se comporte, sous ce point de vue, de manière très proche de celle des rituels romantiques de consommation (cadeaux, voyages, restaurants, films etc.) qui, d'après les enquêtes sociologiques d'Eva Illouz, sont utilisés par les acteurs, même dans leur banalité relative et dans leur instrumentalité évidente, pour accroitre l'intensité émotive des contextes intimes et accentuer la signification (en la contextualisant) de la communication et de l'interaction amoureuse. La figure, pourtant, a l'avantage de la transférabilité et de la réactivation permanente, puisqu'elle n'est pas consommée, mais justement réactualisée et «passée »aux autres dans la répétition du lieu commun (sous la forme d'un morceau de phrase, d'une forme de construction linguistique) qui en constitue le noyau. De plus, il ne s'agit pas d'un medium de projection: elle est déjà toujours disponible en tant que disposition, elle se situe dans le mouvement intentionnel même, sans le symboliser. La figure est ce qu'il y a de commun entre 
des variations multiples - et toujours nouvelles — du thème qui est la figure même, dont le développement historique du discours a établi les contours, tout en soulignant certains traits en dépit des autres ${ }^{5}$. Le symbole-guide n'est pas contraignant: il fonctionne comme une voie grammaticale, résultant d'une histoire discursive et transmettant certaines préférences iconiques ou linguistiques. En ce sens, «pourquoi » et «je-t'-aime» peuvent être une figure aussi bien que « cœur » ou « feuille » (cette dernière renvoyant à la pensée magique de l'amoureux).

Ce caractère figuré, où les figures véhiculent à la fois une tradition pratique partagée et l'intensité émotive individuelle, et mettent «en commun» l'expérience, est la première spécificité du discours amoureux. La définition que Barthes donne du discours étant «toute étendue finie de parole, unifiée du point de vue du contenu, émise et structurée à des fins de communication secondaires, culturalisée par des facteurs autres que ceux de la langue» («La linguistique du discours »612), les figures, la « Topique » amoureuse, constituent l'unité, la cohérence et la (possibilité de) continuité discursive d'un discours dont la deuxième spécificité est justement d'être, avec peu d'exceptions, fragmenté, incohérent du point de vue logique, faible du point de vue argumentatif. C'est justement sur la base de ces caractéristiques constitutives du discours amoureux que Barthes, d'une part, en dénonce la solitude et, d'autre part, offre à ses lecteurs une sorte de traité in vivo d'amour moderne afin de soustraire le discours amoureux à cette solitude.

\section{Le statut du discours amoureux et le projet barthésien}

Au centre de la lecture que Niklas Luhmann a donné de l'évolution de la rhétorique de l'amour passion dans la société européenne occidentale se trouve l'idée voulant que la sémantique du discours amoureux ait une fonction sociale spécifique, et qu'elle ait favorisé la 
naissance de ce que nous connaissons et pratiquons aujourd'hui comme liaisons intimes. Le langage typique de la «folie amoureuse » — développé, pour certains aspects, déjà à partir du XIII $^{\mathrm{e}}$ siècle (par exemple en Italie, dans la poésie du dolce stil novo) — en insistant sur la fatalité de la passion amoureuse, sur l'impossibilité de s'y soustraire par un acte de volonté et sur l'exceptionnalité de la condition d'amoureux, a créé graduellement un espace de relative liberté par rapport aux normes sociales et aux contraintes posées à la sexualité et aux relations personnelles. Développé rapidement en tant que pratique sociale reconnue, forme d'interaction et jeu communicatif spécifique, l'amour (passion) s'affirme autant dans les mœurs que dans la littérature et dans les arts. La sémantique qui le caractérise le rend aussi reconnaissable, identifiable, le circonscrit par des contours visibles mais élastiques (comme ceux du discours), qui lui confèrent un statut propre et reconnu. La méfiance du bon sens commun par rapport au phénomène amoureux, exprimée et renforcée par les adages sur l'amour aveugle, sur l'amour qui conduit à la folie, sur l'amour comme maladie, etc., peut être considérée comme le déploiement psychologique et discursif de cette fonction sociale de recoupement d'un espace pratique, perceptif, d'action et de réflexivité. Au bout d'un long processus historique, cela produit la stabilisation des relations intimes fondées sur l'amour en tant que forme légitime d'union, même dans le sens de l'union conjugale. On observe donc un lien intrinsèque, et à double sens, entre les transformations sociales, psychologiques, linguistiques, littéraires ou artistiques, des pratiques et des mœurs : chaque formation ou transformation du discours, comme Barthes l'affirmait, n'est compréhensible et interprétable que par rapport au contexte extra linguistique de référence, c'està-dire par rapport aux réalités sociales et culturelles dont elle est l'expression et qu'elle contribue en même temps à renforcer et à modifier graduellement. 
Ces observations suggèrent des points de repère pour la description des effets sociaux de l'évolution du discours amoureux, fournissant ainsi des outils théoriques pour la lecture de ses structures et de ses spécificités logiques et grammaticales. Un exemple peut nous aider à comprendre ce double renvoi. Prenons une affirmation légitime dans le discours amoureux, par exemple : «je t'aime, parce que c'est toi », en tant que réponse à la question «pourquoi m'aimes-tu?». La figure «adorable» des Fragments barthésiens, dans sa structure essentiellement tautologique, lui ressemble (est adorable ce qui est adorable; je t'adore, parce que tu es adorable; je t'aime, parce que je t'aime). Ce genre d'affirmations, qui ne décrivent ni n'expliquent rien et qui ne donnent aucune information sur la conduite d'un acteur rationnel, sont pourtant fortement informatives dans le cadre de la communication amoureuse : non seulement elles font avancer la communication amoureuse en tant que telle (elle ont un sens), mais elles le font de manière à gratifier les participants de cette communication amoureuse et à leur donner, pour ainsi dire, l'information convoitée. Or, cette bizarrerie logique, déclinée selon plusieurs variations, a une histoire sémantique, dont l'observation semble la rendre parfaitement compréhensible et plausible au sein du discours amoureux. D'après Luhmann, on assiste à un virage sémantique, autour $\mathrm{du} \mathrm{XVIII}^{\mathrm{e}}$ siècle, pour lequel l'amour même devient la référence fondatrice du discours amoureux, c'est-à-dire que l'amour acquiert un pouvoir justificatif et explicatif par rapport à lui-même: dans le discours amoureux se constitue ainsi une autoreferentialité structurelle. Luhmann suggère d'interpréter ce mouvement sémantique comme le résultat de l'insistance sur l'absence de « raisons » et de justifications de l'amour qui ne soient le fait même d'aimer, d'être amoureux. Si au XVI ${ }^{\mathrm{e}}$ et XVII siècles persiste la tendance à motiver l'amour par les qualités de l'être aimé — et donc à identifier l'amour avec le plaisir de la jouissance des faveurs d'un être plein de beautés et de qualités agréables —, ensuite l'amour 
s'absolutise suivant le mouvement d'une individualisation croissante, qui lie le phénomène amoureux à la personne dans son individualité, ne permettant ni de généralisations ni d'objectivation. C'est en ce sens que le procès de stabilisation de la liaison intime en tant que forme reconnue d'interaction sociale, favorisée par le langage correspondant, accompagne et encourage le procès d'individualisation en Occident. Cette lecture favorise la compréhension du long processus historique qui a produit non seulement l'isolement sémantique progressif du discours amoureux, de la cristallisation de ses bizarreries ${ }^{6}$, mais aussi sa puissance créatrice d'un espace de communication interpersonnelle et de liens ayant un statut reconnu et une structure passible de stabilisation et de légitimation. L'émotion amoureuse, loin d'être limitée au « sentiment », s'articule essentiellement par et dans la communication, se déploie en tant que pratique sémantique, qu'intentionnalité discursive et que base pour certaines typologies des relations sociales.

L'opération de Barthes se fonde justement sur la prise de conscience de la dignité discursive du discours amoureux: non pas une confusion linguistique (et psychologique) passagère, ni une vacillation occasionnelle de l'ordre logique, mais un discours structuré, fort d'une tradition et d'une évolution spécifique, dont l'articulation correspond aux spécificités de l'univers pratique qui lui sert de référence. C'est pourquoi Barthes, en offrant une carte de ce territoire linguistique, retrace patiemment le lien entre disposition ou tentation linguistique subjective (l'amoureux qui parle, en ayant recours à chaque fois à une figure différente) et topique amoureuse surgissant de la tradition littéraire (le Werther de Goethe, Platon, Proust), où les figures se sont formées, où elles ont leurs archétypes, leur scène primitive. Les figures du discours amoureux, qui constituent une «encyclopédie » de la culture amoureuse, peuvent être ainsi rapprochées des lieux communs qui, d'après Barthes, renforcent les dispositions 
linguistiques partagées et produisent, dans la répétition, la communication — dans notre cas, la communication amoureuse ${ }^{7}$.

La dénonciation de Barthes concerne précisément ce point : cette dignité de discours, ayant sa solidité dans la correspondance avec un domaine pratique fortement caractérisé et résultant d'une histoire culturelle richissime, ne paraît plus être reconnue au discours amoureux. Le discours amoureux, nous dit Barthes dans l'introduction aux Fragments, est «ou ignoré, ou déprécié, ou moqué par eux, coupé non seulement du pouvoir, mais aussi de ses mécanismes (sciences, savoirs, arts) ». Le signal le plus clair de cette révocation de dignité, de cette gêne par rapport à la nature intraitable de l'amoureux et du discours auquel il prend part, semble être l'imposition d'un changement de registre et d'une production de sens auxquels l'amoureux est confiné pour assurer sa réconciliation avec la société : l'opinion générale «déprécie toute force excessive et veut que le sujet réduise lui-même le grand ruissellement imaginaire ${ }^{8}$ dont il est traversé sans ordre et sans fin, à une crise douloureuse, morbide, dont il faut guérir [...] l'histoire d'amour est le tribut que l'amoureux doit payer au monde pour se réconcilier avec lui » (Fragments 463). C'est pour décourager cette attitude constante de l'Autre social par rapport aux manifestations du phénomène amoureux, pour opposer une résistance à la tendance réductive qui reconduit ces manifestations à l'histoire d'amour ou à un épisode symptomatique, que Barthes choisit un « ordre absolument insignifiant» (l'ordre alphabétique) pour présenter les figures dans les Fragments. D'une part, il dénonce alors l'indifférence et le mépris du pouvoir et de ses langages par rapport au discours amoureux; d'autre part, il met en garde contre toute réduction de celui-ci à une folie momentanée, à un bouleversement de langage dépourvu non seulement de raison, mais de sens, puisqu'il ne se conforme pas aux formes explicatives, argumentatives, fondatrices, délibératives des discours sociaux du sens commun, de l'instrumentalité, de la 
production, du savoir au sens strict, etc. Dans un fameux entretien avec Philippe Roger, paru dans Playboy en septembre 1977, Barthes affirme que le discours amoureux est déprécié par le langage populaire, qui assimile l'amoureux «à un lunaire, à un fou»; mais surtout par les «langages théoriques »: «ou bien ils n’en parlent pas du tout, comme le langage politique, le langage marxiste. Ou bien ils en parlent avec finesse, mais d'une façon dépréciative, comme la psychanalyse ». À la question de Philippe Roger, l'interrogeant sur les raisons de cette dépréciation, Barthes répond : «L'amour-passion (celui dont j'ai parlé) n’est pas “bien vu”; on le considère comme une maladie dont il faut guérir; on ne lui attribue pas, comme autrefois, un pouvoir d'enrichissement » (Barthes, « Entretien avec P. Roger » 406).

L'offensive barthésienne consiste donc en une opération qui présuppose une profonde réflexion au sujet de la structure et des spécificités du discours amoureux — avec la psychologie correspondante en tant que trace incorporée d'une histoire culturelle —, et qui, en laissant place à l'affirmation du discours amoureux en tant que tel, vise un double effet. En premier lieu, il s'agit de soustraire le discours amoureux à toute tentative réductionniste par le dévoilement de la tentation toujours présente de le réduire à une symptomatologie, tentation qui est un effet de déligitimation du cadre du discours amoureux par d'autres domaines de pratique sociale. En deuxième lieu, et par le même geste, il s'agit d'offrir un espace, une «occasion» de reconnaissance de l'expérience, de réflexivité, à tous ces sujets inconnus, hypothétiques, qui pratiquent le discours amoureux, mais sans qu'on parvienne à le savoir.

En réponse à Philippe Roger qui lui demande comment cet amoureux difficilement visible, puisque « déprécié » partout, est reconnaissable dans notre quotidien, Barthes répond :

Perfidement, je dirai que j'ai écrit le livre pour pouvoir le reconnaître! [...] Il ne se reconnait pas extérieurement. Parce que, dans la vie urbaine actuelle, il n'y a plus aucune des poses du pathétique amoureux. [...] On n'a même plus la morphologie des traits de 
l'amoureux, ses expressions, sa mimique; alors qu'au XIX ${ }^{\mathrm{e}}$, il y avait des centaines de lithographies, de peintures, de gravures, qui le représentaient. Donc, on ne peut plus reconnaître un amoureux dans la rue. Nous sommes entourés d'êtres dont nous ne pouvons pas savoir s'ils sont amoureux. Car, s'ils le sont, ils se contrôlent énormément. (406)

Les contraintes appliquées au discours amoureux produisent, ainsi, de nouvelles postures des amoureux, attitudes psychologiques et corporelles qui, on peut le supposer, exercent un effet de retour sur les émotions mêmes. En ce sens, et comme nous l'avons déjà souligné, offrir un espace de reconnaissance de l'expérience et de l'émotivité signifie offrir, par une intervention sur les limites et les espaces du discours, un cadre d'épanouissement émotif, psychologique et actif.

Encore dans le même entretien, Barthes corrige la question de P. Roger, qui lui demandait si son livre était militant, en disant que les Fragments comportent plutôt une position de valeur, ainsi qu'une morale, « une morale d'affirmation. Il ne faut pas se laisser impressionner par les dépréciations dont le sentiment amoureux est l'objet. Il faut affirmer. Il faut oser. Oser aimer... » (417). C'est en ce sens qu'il faut lire l'allusion de Barthes à une hypothétique communauté, ou plutôt une association (ce qui évoque justement une prise de conscience et une forme d'affirmation) d'amoureux, à laquelle le livre s'adresserait: «Le livre, idéalement, serait une coopérative : “Aux Lecteurs - aux Amoureux - Réunis" »(Fragments 30). La constitution patiente de cette communauté se réalise par la circulation libre et, on pourrait dire, enthousiaste des figures du discours amoureux (dont Barthes ne donne qu'une sélection exemplaire) : la communauté de discours ne se constitue pourtant pas grâce à une forme de transférabilité générale de l'information ou du savoir transmis par la figure, mais plutôt par une reconnaissance (qui est immédiatement une expérience) de la disposition linguistique correspondant au geste qu'est chaque figure. Celle-ci donne l'occasion de reconnaître une capacité et une disposition 
personnelles, peut-être cachées, oubliées, latentes ou refoulées, et ainsi une proximité psychologique et d'expérience avec les autres « Amoureux ».

On pourrait dire que le but des Fragments est d'encourager ce jeu communicatif, dont le rythme est scandé par l'alternance entre le geste d'offrir la figure, qui en même temps la réactive, et celui de la recevoir, à son tour une revivification de son potentiel. C'est ainsi que l'opération de Barthes révèle sa portée morale, et même politique : restituer une légitimité et une écoute à un cadre entier de production communicative, de réflexivité, de partage - un cadre qui se constitue par ce même geste de partage, par la «performance» affirmative barthésienne du discours amoureux. Dans un entretien avec Laurent Kissel, paru dans Le Figaro littéraire le 5 juillet 1975 (soit deux ans avant la parution des Fragments), Barthes explique, tout en soulignant la différence essentielle entre discours amoureux et écriture amoureuse :

Le discours, c'est un ensemble de figures, c'est-à-dire de configurations de raisonnements, d'images qui sont à cheval entre un contenu et une forme, mais ce n'est pas du pur signifiant comme dans l'écriture. Le discours amoureux, c'est une structure d'énonciation où se rassemblent les différentes possibilités qu'a le sujet humain pour se placer dans ce qu'il dit. On peut alors se demander si ce discours est anachronique ou non. Je ne crois pas qu'il le soit, mais, actuellement, il est censuré par la mode. On retrouve ce discours amoureux, soit dans des productions populaires, comme les chansons, la musique pop'..., soit d'une façon clandestine chez un certain nombre de sujets, mais, par définition, on ne le sait pas. La culture d'avant-garde semble proscrire ce discours, mais il existe. [...] Il y a eu tout un ensemble de siècles où ce discours a trouvé son expression et où il était ressenti comme d'une très haute valeur. (Barthes, «Roland Barthes met le langage en question » 917)

La valeur du discours amoureux est celle de l'expérience de la passion, avec sa propre démesure et sa propre productivité, son importance au niveau de l'organisation subjective de l'expérience, de l'articulation de soi par rapport à l'autre, de l'ouverture d'une dimension imaginative et énergétique nouvelle, de la découverte d'un potentiel subjectif créateur. L'allure et les effets positivement subversifs de l'amour passion créent des liens, des possibilités, des 
valeurs, des engagements, des attitudes, des formes de courage, de respect et d'adoration, des espaces de vision inimaginables en dehors de son cadre d'influence. La proximité traditionnelle du discours amoureux et de l'expression artistique et littéraire — une proximité à son tour désormais démodée, comme Barthes le souligne — nous donne la mesure de la productivité de cette soudaine et profonde transformation de l'expérience subjective. Tout point de vue externe qui ne soit pas disponible à l'écoute de cette spécificité en tant que telle finit par la forcer au silence, à une certaine forme de pudeur, même de honte.

\section{Qu'est-ce que signifie répondre à l'appel de Barthes?}

Au début de son émission Répliques, consacrée au Paradigme de l'amour (France Culture, 9 oct. 2010), Alain Finkielkraut citait l'affirmation de Roland Barthes qui constitue le noyau et le point de départ du travail ici présenté. Aujourd'hui, observait pourtant Finkielkraut, la situation paraît avoir changé : le discours amoureux semble être pratiqué partout, voire «pris en charge par la pensée », comme en témoigneraient, d'après Finkielkraut, les ouvrages de ses deux interlocuteurs dans l'émission en question, Pascal Bruckner et Luc Ferry. Et, on peut l'imaginer, comme en témoignerait aussi l'émission même, consacrée au Paradigme de l'amour.

Après avoir écouté l'émission, il est difficile de s'empêcher de se demander si c'est bien le discours amoureux tel qu'il est «parlé par des milliers d'individus » (à ce discours-ci, pensait Barthes dans son introduction aux Fragments) que ces penseurs ont pris en charge, pour le soutenir, pour le reconduire à l'actualité qui, d'après Barthes, le tenait à l'écart. Y a-t-il de la part de la philosophie et du discours savant un véritable effort d'écoute et de compréhension de ce que c'est que s'aimer, parler d'amour dans la société contemporaine, ou bien le malentendu qui 
se méprend sur la différence entre discours et métadiscours amoureux serait-il toujours à l'œuvre? Avons-nous commencé le travail de prise en charge souhaité par Barthes?

Les points fondamentaux de la discussion entre Finkielkraut, Bruckner et Ferry sont la révolution introduite, surtout au XIX ${ }^{\mathrm{e}}$ siècle, par l'idéal de l'amour romantique et par la diffusion du mariage d'amour (remplaçant le mariage arrangé) et ses conséquences sur l'institution du mariage même — ou mieux, sur la stabilité du lien conjugal (même sous d'autres formes, comme le PACS). Concernant le premier point, dans son dernier ouvrage (La révolution de l'amour), Luc Ferry souligne la portée révolutionnaire de l'union familiale moderne fondée sur l'amour et sur le sentiment (y compris l'amour «passionné » pour les enfants, acquisition historiquement assez récente). L'effet éducateur ou éthique de cette révolution, apprenant aux individus l'attention pour l'autre, la sympathie et l'amour pour l'humain, aurait créé les conditions pour un nouvel humanisme. Sans contester la portée novatrice du passage au mariage d'amour, les deux autres interlocuteurs y reconnaissaient plutôt des signes de décadence. On en vient ainsi au deuxième et principal point de la discussion: d'après A. Finkielkraut, le mariage d'amour efface la distinction, respectée par les Anciens et dont nous ne voulons plus, entre amour conjugal et amour passion. Ainsi, l'on doit faire face au problème majeur de la stabilisation du couple après que l'amour passionné, inévitablement, cesse. De plus, ajoute P. Bruckner (auteur en 2010 de Le mariage d'amour a-t-il échoué?), dans le monde actuel, hanté par le mythe de la passion, tout plaidoyer pour un retour à l'amour conjugal, conciliant cœur et raison, sensible envers l'individu et non seulement envers l'idéal, est traité de «réactionnaire» et de «rabat-joie». Pourtant, ajoutent Ferry et Finkielkraut, seules les valeurs traditionnelles seraient susceptibles d'endiguer la dérive d'appauvrissement de l'amour, réduit à la passion et surtout, dans la société capitaliste, à la consommation. Pour terminer, Finkielkraut explique le «succès du PACS » par la diffusion 
d'un rapport de consommation à l'expérience amoureuse, par le «zapping amoureux », par le refus de l'engagement, par un souci de confort dans la démarche de séparation, celle-ci étant considérée comme inévitable, puisque l'amour (passion) ne dure pas. C'est la formule même du PACS, nous dit Ferry, que de prévoir la possibilité du «zapping amoureux » : seulement le choix durable, l'engagement, l'«amour-action» pourraient nous éviter de changer de partenaire comme on change d'iPhone ou de Blackberry. Là où l'éducation sentimentale est prise entre idéalisation romantique de l'amour passion et désacralisation de la sexualité (voire diffusion indiscriminée de la pornographie), la solution serait le retour à la raison en amour (contre la «passion », selon la polarité raison/passion).

On peut commencer par expliciter certaines prémisses discutables dans les affirmations que je viens de résumer. Tout d'abord, l'état amoureux passionné s'y trouve pris dans les mêmes mécanismes de dépréciation et de réduction dénoncés par Barthes. D’une part, il semble être considéré comme un amour d'une ligue inférieure par rapport à l'amour de « raison » ou à l'amour conjugal; d'autre part, il semble être traité comme une sorte d'aveuglement ou de fixation du sujet passionné, voire un caprice des individus contemporains, paresseux et immatures par rapport à l'engagement demandé par l'union amoureuse conjugale. Or, c'est précisément à cet appauvrissement préjudiciel de l'expérience amoureuse que Barthes essayait de répondre.

Un deuxième point discutable concerne, par conséquent, l'attribution à l'amour passion d'un certain degré d'inauthenticité. Son inconstance, ses éclats, ses humeurs, ses bouleversements, ses manifestations idiosyncratiques sont considérées comme des formes de corruption (momentanée, à dépasser) de l'amour «authentique » (l'amour « conjugal»?). Son caractère hétérogène par rapport aux assomptions de responsabilité morale, sa méfiance par 
rapport à toute instance de sanction qui ne soit la puissance de l'amour même, lui attirent un préjugé de superficialité. Attitude constitutivement réfractaire à la rationalisation et à la psychologie propre de la justification morale et de l'engagement éthique, l'amour passion est soupçonné, de manière moraliste, d'une inauthenticité fondamentale, à cause de laquelle il doit être dépassé et transformé afin de créer les conditions de stabilité de l'union.

En troisième lieu, des présuppositions normatives très fortes sont imposées au débat par une certaine idée de ce que cette union stable doit être, ainsi que de son sens moral. Une forme déguisée de condamnation morale semble peser encore sur la rupture d'un mariage ou d'une union, comme si elle était la marque d'une sorte de manque de sérieux moral en amour. Cela présuppose que la durée de toute une vie fasse essentiellement partie de la compréhension éthique du mariage. En ce sens, et en quatrième lieu, d'autres formes d'union et de partnership (dont le PACS) manifesteraient une jouissance irresponsable et superficielle du lien amoureux : leur succès témoignerait d'une tendance à la «consommation», au «zapping » amoureux. Ensuite, ce retour souhaité à l'authenticité de l'amour conjugal (ou amour-raison), contre la dérive de «corruption » de l'amour, part de la présupposition (métaphysique), qu'il existe une forme authentique d'expérience d'amour permettant une taxonomie morale des pratiques amoureuses, indépendamment de l'époque et du contexte social dont on discute. Enfin, cela prétend également qu'il suffise un retour à cet « originaire » authentique — dont on présuppose qu'il correspond à la « nature » humaine — pour remettre en ordre la confusion dans l'espace amoureux.

La liste de ces prémisses discutables pourrait continuer. Mais revenons à la question centrale : la discussion mentionnée se met-elle à l'écoute du discours amoureux tel qu'il est parlé par les individus qui (s')aiment, aujourd'hui? Valorise-t-elle l'expérience amoureuse dans sa 
richesse et sa complexité, si souvent refusées par la société et par les autres discours? Cette description des pratiques amoureuses reflète-t-elle leur réalité (et leur réflexivité) hic et nunc?

À bien y regarder, la solution proposée d'un retour à l'engagement éthique n'est pas du tout nouvelle. À la recherche d'une recette pour résoudre le problème de l'instabilité de l'amour, elle finit par répliquer en réalité le schéma qui oppose passion et raison, jouissance et renonciation, individualisme et engagement. Il s'agit du même schéma qui influence en partie le discours amoureux, qui caractérise son évolution historique, qui crée le malaise généralisé dont l'expression amoureuse de la passion finit par souffrir à l'époque contemporaine. Il s'agit du même schéma, et c'est l'hypothèse interprétative que je propose, qui constitue le point de repère principal des transformations en cours dans les pratiques amoureuses et les formes de liaisons. Pour paraphraser Watzlawick et al., il s'agirait ici d'une occurrence de cette forme de problème qu'ils définissent comme more of the same, c'est-à-dire dans laquelle le problème est constitué par la solution envisagée.

Le schéma moraliste qui sert de base à la solution proposée (amour-raison contre dérèglement de la passion) se nourrit donc de l'opposition traditionnelle entre amour passion et amour conjugal, entre désordre, instabilité, inconstance et stabilité bien rangée, durable. C'est le même schéma qui caractérise toute la tradition occidentale de la sémantique amoureuse, et par lequel la condamnation morale a pesé sur l'amour passion et sur son inaptitude à fonder des relations durables et morales - en lui garantissant pourtant, nous l'avons vu, un espace de liberté et de développement, qui l'a finalement conduit à une légitimation au cœur de la vie conjugale à l'âge romantique. Ce schéma fait donc partie du patrimoine de «savoir » enregistré dans le discours amoureux traditionnel, dans l'imaginaire amoureux et dans les dispositions linguistiques correspondantes. Il est déjà actif dans les catégories de compréhension et de 
maîtrise de l'expérience, dont on souhaiterait une transformation par une réaffirmation de l'opposition qui fonde ce schéma. More of the same.

Il serait légitime de se demander aussi si ce même langage opposant de manière rigide le libertinage à l'engagement, la tentation à l'ascèse, le désir à la moralité, n'est pas l'allié parfait du discours de la consommation, au lieu d'être son ennemi : il légitime notamment le langage de la tentation irrésistible, du «petit péché », du «se-faire-plaisir» qui anime la diffusion publicitaire des biens de consommation. Ainsi, selon la leçon de Foucault, cet apparent dérèglement du désir et ce retour de la passion déchainée ne le seraient point.

De plus, ce schéma semble être à la base de la transformation en cours par rapport aux formes de l'union conjugale, transformation qui porte l'identification romantique de l'amour et du mariage à ses conséquences logiques : une fois que l'amour (passionné?) finit, finit également l'union conjugale. En ce sens, le mariage d'amour — ou l'union durable fondée sur l'amour en général - aurait «échoué » si on comprend le mariage en tant qu'institution ayant la durée de toute une vie. Si son but est au contraire celui de créer un cadre pour la tentative — individuelle, c'est-à-dire articulée par chaque relation ou chaque couple à sa manière — de stabilisation de l'amour passionné, alors le mariage d'amour n'a pas échoué. Certes Finkielkraut, Bruckner et Ferry, tout comme beaucoup d'autres penseurs, ont raison de mettre en évidence la méfiance croissante par rapport à l'engagement à long terme, la tendance des individus à retarder le moment du choix. Cela n'est pourtant qu'un aspect de l'expérience amoureuse actuelle, qui comporte, il me semble, un autre paradigme éthique, comme on le verra dans la suite. Cela pourrait être attribué tout également à l'effet des fausses catégories de compréhension des possibilités d'expérience amoureuse, notamment celles qui imposent l'alternative entre (exaltation de la) passion et (platitude de la) vie conjugale. Peut-être que cette solution perd de 
vue les enjeux plus profonds de notre époque, tout comme les transformations historiques de la société, de la subjectivité et du discours qui les relie?

\section{Un nouveau cadre de compréhension de l'expérience amoureuse}

On reconnaît dans le paradigme historique de l'amour romantique la stabilisation de l'amour pour l'individu singulier en tant que liaison stable et privilégiée avec celui-ci. La construction d'un lien intime entre deux existences personnelles (projet existentiel, « sens » de la vie, communication, attentes par rapport à la relation, etc.), apparemment « naturelle » dans notre expérience amoureuse, est un stade assez récent (même pas vieux de deux siècles) de l'évolution des pratiques amoureuses occidentales: cela présuppose des transformations majeures et progressives de la psychologie individuelle, par exemple le développement de la capacité à concevoir sa propre individualité, celle d'autrui et des formes de relation entre les deux. Voilà une autre manière de décrire la révolution du mariage d'amour, composante essentielle du discours romantique. Ses effets, comme le souligne Luc Ferry, sont remarquables.

Concernant la relation entre imaginaire amoureux, discours amoureux et subjectivité, A. Giddens observe que l'amour romantique introduit la narration dans l'existence individuelle, conférant à celle-ci un horizon de sublime. Le moi et l'autre font partie d'une narration (romance) qui transfigure la vie individuelle, ainsi que la liaison d'amour devient relation d'amour, histoire d'amour — liée également à l'imaginaire romantique du destin, de l'affinité des âmes, de l'amour comme valeur absolue. Dans les mots de Giddens, «romantic love presumes some degree of self-interrogation. How do I feel about the other? How does the other feel about me? Are our feelings "profound" enough to support a long-term involvement? » (Giddens 44). C'est donc à une forme spécifique de réflexivité, à un certain discours sur la 
trajectoire personnelle, sur le bonheur individuel, sur la réalisation de soi, que l'idéal de l'amour romantique est lié (il suffit de jeter un coup d'œil sur son «manifeste », à savoir la Lucinde de F. Schlegel). À son tour, Luhmann soulignait que l'évolution de la sémantique amoureuse en Occident est liée comme en double spirale avec le procès d'individualisation. Le passage historique du modèle de l'amour passion à celui d'amour romantique, et de ce dernier à celui de la partnership, à l'intimacy ou la «pure relationship» (Giddens), requiert d'être lu non seulement à partir des modifications sociales, mais aussi à partir des changements intervenus dans les cadres de l'expérience, de la réflexivité, dans les modalités et les formes du projet de vie individuelle, conséquemment dans la communication sociale et intime et dans la structure des relations qui en dérivent. À son tour, chaque étape de la sémantique amoureuse occidentale c'est-à-dire chacune des formes de compréhension et de pratique disponibles dans l'histoire de la civilisation occidentale pour donner une place aux pratiques amoureuses dans la vie individuelle et sociale — produit des effets de retour (d'où l'idée de la double spirale) sur l'articulation de l'individualité, ainsi que sur la structure sociale de référence. Une critique des formes contemporaines de pratique amoureuse ne peut en aucun cas faire abstraction de cela : elle est obligée, comme le montrent les grandes analyses d'A. Giddens, de N. Luhmann, d'E. Illouz, de U. Beck et d'E. Beck-Gernsheim, de tenir compte du contexte culturel et social dans lequel elles s'inscrivent. À ce propos, une référence en passant à la société de la consommation ne paraît absolument pas suffisante.

Considérer les individus contemporains comme les victimes inconscientes du discours de la consommation, ou bien d'un discours vulgarisé sur l'amour passion et sur le romantisme, c'est non seulement oublier la dépendance mentionnée, mais surtout négliger d'observer la complexité des cadres et des catégories de la pratique amoureuse dans notre société. Eva Illouz, qui a 
conduit justement une enquête sur le rapport des acteurs sociaux, dans leur vie amoureuse et relationnelle, à l'idéal romantique qui colonise l'imaginaire médiatique et publicitaire de la société capitaliste occidentale, montre que ce rapport est bien plus complexe et ambivalent qu'il n’y paraît: les entretiens qu'elle a menés dévoilent une méfiance généralisée, bien que proportionnelle au statut social et au niveau d'instruction, par rapport à la capacité de l'idée d'amour romantique à stabiliser une liaison durable. Loin d'impliquer un retour à des formes anachroniques d'amour «conjugal», ce cadre montre la tendance à une révision — dont il est même possible, si l'on y prête attention, de décerner les signes et les cadres discursifs, ainsi que les structures de réflexivité, dans le tant méprisé discours «vulgarisé » des magazines — à la fois du lien conjugal et de l'héritage romantique.

$\mathrm{Au} \mathrm{XX}^{\mathrm{e}}$ siècle, on assiste à l'inclusion structurelle dans l'expérience et dans la réflexivité amoureuse de deux composantes novatrices par rapport aux étapes précédentes : d'une part (Luc Ferry y insiste avec justesse), la compréhension de l'autre en tant qu'individu, de sa vision du monde, de ses valeurs etc.; d'autre part, la conscience de la nature problématique et conflictuelle de la relation amoureuse — voire la pratique de résolution de problèmes relationnels comme activité fondatrice du couple et du lien amoureux. On assiste à l'intégration consciente et constitutive, dans la vie amoureuse, du problème de la recherche d'une solution pratique à la question de la stabilisation de l'union fondée sur l'amour ${ }^{9}$ — question qui, d'après Finkielkraut et Brucker, serait négligée par la pratique amoureuse actuelle. Au lieu d'être la démonstration de cette négligence présumée, le PACS et les formes de relation idéologiquement différentes du mariage semblent refléter cette intégration structurelle, dans l'expérience amoureuse, des aspects problématiques et de l'instabilité de la relation. C'est dans ces formes novatrices que s'exprime, autrement dit, la double révision mentionnée ci-dessus, à la fois de l'idéal de l'amour romantique 
(puisqu'on n'est plus disposé à une confiance aveugle par rapport à l'idiosyncrasie du sentiment, d'où l'hésitation répandue à y fonder de manière exclusive l'engagement dans la relation stable) et du mythe du mariage, voire de l'amour conjugal (d'où l'hésitation à considérer la relation conjugale ou le partnership stable comme censés avoir — par idéologie, je dirais — la durée d'une vie entière).

Classer le PACS et les formes de relation amoureuse différentes du mariage traditionnel comme un choix de confort, de commodité, comme l'inscription du «zapping amoureux » dans la reconnaissance sociale et institutionnelle, c'est oublier le retour réflexif qui, sur une échelle sociale et culturelle, a été causé par l'expérience et par la prise de conscience de l'instabilité non pas de l'amour passion, mais de la relation intime fondée sur le sentiment en général. La fragilité des relations personnelles augmente de manière directement proportionnelle à l'investissement individuel sur la relation : aussitôt que celle-ci devient un projet totalisant de vie, et qu'elle consiste en la conciliation et en l'union de deux existences individuelles dans l'amour, elle est chargée d'attentes, d'intentions, de représentations (à divers niveaux de réalité) et donc — à côté des joies et des gratifications — de frustration, de déceptions grandes et petites, qui se reflètent dans la communication quotidienne et dans la transformation que la relation même produit chez les individus impliqués (Giddens en donne des exemples très représentatifs).

L'intégration dans la relation intime de la capacité de comprendre et de connaître l'autre dans son individualité, la profondeur d'intimité, de complicité, présupposent une capacité réflexive d'autoanalyse et d'introspection, mais aussi un fort investissement sur l'autoréalisation, sur le souci de soi, sur la recherche du bonheur individuel — et sur la présence de critères spécifiques pour juger de ce «bonheur » et du degré de satisfaction dans l'existence personnelle. Si la relation amoureuse conjugale doit soutenir «par amour » l'épanouissement (paritaire) de 
chacun de ses membres, alors le bonheur individuel est un critère fondamental de sa réflexivité : en ce sens, comme Amélie Rorty le soulignait, cette demande conjointe de profondeur, de réciprocité et de continuité crée les conditions de l'intimité romantique, mais aussi de la possibilité de sa dissolution.

\section{Pour conclure : potentiel éthique des formes de relation contemporaines}

Il n'est pas difficile, de nos jours, de trouver des exemples de la levée de boucliers contre les formes contemporaines de multi-expérience relationnelle, contre les effets désastreux de la désillusion amoureuse en termes de refus du lien durable, d'hésitation dans l'engagement, de fuite dans l'idéal, etc. J'espère avoir montré en quoi cette lecture et cette attitude moralisatrices ne rendent justice ni aux transformations de la subjectivité amoureuse contemporaine, ni aux tendances dominantes dans l'espace des pratiques amoureuses (surtout chez les jeunes générations). En d'autres termes, c'est le discours amoureux tel qu'il a lieu à notre époque

qu'elles finissent par manquer. Équipée avec les outils du sens commun et de son regard moralisateur, cette lecture se contente de considérer les transformations en cours comme la redoutable corruption d'un idéal d'amour, voire de la morale liée à cet idéal d'amour (l'amour conjugal, scilicet : le mariage bourgeois).

En dépit de l'accusation de superficialité, poser le lien sentimental sous la condition de la satisfaction des parties impliquées, du bonheur individuel et partagé dans la relation, signifie en prendre sur soi la responsabilité envers soi-même et envers l'autre, de manière réaliste et respectueuse. Si ce qu'on souhaite est une forme de rationalité et de sagesse en amour, il faut admettre qu'une telle tendance, forte de l'accumulation des données d'expérience de plusieurs siècles de pratiques amoureuses, en fait en grande mesure la preuve. Ces changements d'horizon, 
d'imaginaire et de formes d'engagement amoureux correspondent, de plus, à des changements sociaux très profonds, qu'on ne peut pas négliger. La parité et l'égalité croissante, ainsi que la demande croissante de parité entre les deux sexes dans les relations amoureuses, font qu'aujourd'hui deux projets de vie — comprenant réalisation personnelle, professionnelle et sentimentale ou familiale — doivent être harmonisés dans la relation intime. Le fait que les femmes se soustraient de plus en plus au rôle de centre de gravité de l'union conjugale (en tant que médiatrices, en tant que présence domestique stabilisatrice, en tant que «compensatrices » des tendances centrifuges traditionnellement attribuées au désir masculin ${ }^{10}$ ), et qu'elles revendiquent de manière de plus en plus naturelle, de moins en moins moralisatrice leurs espaces d'épanouissement, leur indépendance et leur distance par rapport aux rôles traditionnels, augmente l'instabilité du lien, son irritabilité. En ce sens, c'est aussi par rapport aux conquêtes du mouvement d'émancipation de la femme que la mention de l'amour conjugal a un goût décidément anachronique.

Encore sur ce point, on trouve des indices des changements en cours, par exemple, dans les médias, sismographes très sensibles face aux tendances du discours amoureux actuel, qu'ils doivent capter afin de s'adresser à leur potentiel public tout en en détectant l'esprit. Un magazine italien suggérait récemment que les rôles que les jeunes femmes d'il y a une vingtaine d'années auraient considérés comme typiquement masculins et féminins dans les phases initiales d'une relation sont manifestement en train de s'hybrider — les femmes paraissant être moins disposées à l'idéalisation et à la stabilisation rapide de la relation, etc. ( $c f$. Soncini).

C'est dans ce même cadre, celui du discours amoureux courant, qu'on peut chercher les indices et les matériaux pour comprendre en détail les phénomènes qui se déploient de nos jours dans les pratiques amoureuses — pour lire, par exemple, la déconstruction pratique de l'idéal du 
mariage bourgeois (dans sa normativité liée à l'indissolubilité et à l'exclusivité) et de la morale correspondante qui fondait à la fois le mariage bourgeois et l'amour romantique; et pour décerner les liens entre ce procès-ci et les transformations des identités de genre dans leur articulation pratique quotidienne. Quelles figures, quels signes anciens et nouveaux, quels lieux communs se répètent dans le discours amoureux contemporain? En quoi les nouvelles formes de communication (social networks, chats, smart phones, VoIP, etc.) influencent-elles l'autoreprésentation, les dispositions langagières, la réflexivité sur les émotions? En quoi les structures économiques et de production se reflètent-elles dans les conditions et les formes de la vie affective intime? Quels jeux de distance et de rapprochement, d'intensité et de détachement, quelles revendications et quelles craintes — quelles dynamiques de pouvoir ${ }^{11}$ s'expriment dans les spécificités du discours amoureux, si ce dernier n'est ni le délire dépourvu de tout sens d'individus isolément « pathologiques », ni un caprice singularisé, mais plutôt un lieu complexe et multidimensionnel d'interaction entre pressions morales et sociales, héritages historiques, sédimentations culturelles et l'expérience émotive et communicative singulière?

Au lieu de considérer que tout abandon de l'éthique conjugale signifie un refus de l'engagement éthique dans le lien sentimental; que toute hésitation par rapport au type d'engagement monogamique caractérisant la morale du mariage bourgeois signifie une décadence des pratiques amoureuses, un «manque »d'amour; que toute relation limitée dans le temps et toute forme de non-exclusivité amoureuse soient moralement redoutables, on pourrait se demander si nous n'assistons pas à la recherche pratique d'une autre forme d'amour et d'une autre compréhension de sa dimension morale. S'il est vrai, comme Nietzsche l'affirme, qu'on peut changer les choses en leur attribuant de nouveaux noms, une philosophie soucieuse de soutenir le discours amoureux des sujets qui le parlent, d'améliorer leur compréhension d'eux- 
mêmes et du monde, finalement de favoriser la création des conditions pour une vie amoureuse plus heureuse, productrice et gratifiante, pourrait commencer en se mettant à l'écoute.

\section{Notes}

* La recherche dont ce travail a été issu a été rendue possible par la Käthe-Kluth Fellowship de la Ernst-Moritz-Arndt-Universität de Greifswald. Ma profonde reconnaissance va à Martin Breaugh pour l'aide dans la révision de ce travail.

${ }^{1}$ Barthes tint un séminaire sur le discours amoureux à l'École pratique des hautes études en 1974-1976, dont les notes ont été publiées en 2007 au Seuil. Destinées à l'enseignement, ces notes de cours sont conçues et organisées de manière très différente par rapport aux Fragments, qui sont, on le verra, une œuvre engagée pour un public plus large. Sur le rapport entre enseignement, écriture et parole dans son œuvre, voir l'explication de Roland Barthes dans l'«Entretien » avec Alain Poirson (543 sq.).

${ }^{2}$ Ainsi Mauss : «J'ai donc eu pendant de nombreuses années cette notion de la nature sociale de 1" "habitus". [...] Le mot traduit, infiniment mieux qu"habitude", 1'"exis", 1'“acquis" et la "faculté" d'Aristote (qui était un psychologue). Il ne désigne pas ces habitudes métaphysiques, cette "mémoire" mystérieuse, sujets de volumes ou de courtes et fameuses thèses. Ces "habitudes" varient non pas simplement avec les individus et leurs imitations, elles varient surtout avec les sociétés, les éducations, les convenances et les modes, les prestiges. Il faut y voir des techniques et l'ouvrage de la raison pratique collective et individuelle, là où on ne voit d'ordinaire que l'âme et ses facultés de répétition» (366 sq.).

${ }^{3}$ Mauss donne l'exemple du «sang froid », celui-ci étant un mécanisme de retard, inhibition de mouvements désordonnés, qui permet une réponse somatique coordonnée dans la direction du but choisi. Il s'agit d'une technique de résistance à l'émoi, par la maîtrise des réactions musculaires, et qui, par cela, fait du corps et des émotions liées à sa posture l'instrument principal pour l'exécution d'activités, stratégies et attitudes psychologiques complexes dans l'interaction sociale.

4 Et ceci bien qu'au cœur de la figure, comme on le verra, ne se trouve souvent qu'une proposition inachevée et dépourvue d'intelligibilité complète : "Au fond de chaque figure gît une phrase, souvent inconnue (inconsciente?), qui a son emploi dans l'économie signifiante du sujet amoureux. Cette phrase mère (ici seulement postulée) n'est pas une phrase pleine, ce n'est pas un message achevé. Son principe actif n'est pas ce qu'elle dit, mais ce qu'elle articule : elle n'est, à tout prendre, qu'un "air syntaxique", "un mode de construction". Par exemple, si le sujet attend l'objet aimé à un rendez-vous, un air de phrase vient à ressassement dans la tête : "Tout de même, ce n'est pas chic..."; "il/elle aurait bien pu..."; "il lelle sait très bien pourtant..." 
Pouvoir, savoir quoi? Peu importe, la figure "Attente" est déjà formée » (Barthes, Fragments $31)$.

${ }^{5}$ Les figures constituent ainsi une sorte de Topique amoureuse : «ce code, chacun peut le remplir au gré de sa propre histoire; maigre ou pas, il faut donc que la figure soit là, que la place (la case) en soit réservée. C'est comme s'il y avait une Topique amoureuse, dont la figure fût un lieu (topos). Or, le propre d'une Topique, c'est d'être un peu vide : une Topique est par statut à moitié codée, à moitié projective (ou projective, parce que codée) » (Barthes, Fragments 30).

${ }^{6}$ Déjà dans le Banquet platonique, d'après Barthes, le discours amoureux abandonne, par la palinodie socratique, le régistre de la science de l'amour pour devenir «éloge »: «tout discours sur l'amour recèle un éloge de l'être aimé ou désiré (ou refusé) » (Barthes, Discours amoureux 333). Paul Ricoeur retrouve déjà cette structure caractéristique d'hymne et de louange dans le Cantique des cantiques.

${ }^{7}$ F. de Ruggieri $(511$, n. 1) souligne que le lieu commun moderne n'est pas répétition d'une forme, mais d'un contenu, distinction très importante pour notre analyse du partage des pratiques et des expériences par le discours amoureux. $C f$. Barthes, « Il luogo comune ».

${ }^{8}$ Dans l'entretien cité avec Alain Poirson, Barthes précise, à propos des Fragments : «j'entends imaginaire, non au sens de l'Imagination, mais dans le sens assez technique que lui donne Lacan: un registre psychique, différent du Symbolique et du Réel, qui est caractérisé par l'adhérence très forte du sujet à une image » (542).

9 Luhmann définit cela comme «Programm des Verstehens» («programme de la compréhension » ou « programme du comprendre »).

${ }^{10}$ Dans le lieu commun de «l'homme chasseur» (voir aussi la figure «Absence» dans les Fragments de Barthes), dans la naturalisation oubliée d'un préjugé culturel (et des implications complémentaires pour l'autre sexe), il y a aussi l'affirmation implicite d'un droit naturel à une certaine sexualité et à une certaine émotivité — ce qui implique la révocation de ce droit pour le sexe complémentaire, c'est à dire pour la « féminité ».

11 Ainsi que Barthes le décrivait dans sa «Leçon» inaugurale au Collège de France en 1978 : « le pouvoir est le parasite d'un organisme trans-social, lié à l'histoire entière de l'homme, et non pas seulement à son histoire politique, historique. Cet objet en quoi s'inscrit le pouvoir, de toute éternité humaine, c'est : le langage — ou pour être plus précis, son expression obligé : la langue. Le langage est une législation, la langue en est le code $[\ldots]$ : toute la langue est une rection généralisée » (431). 


\section{Ouvrages cités}

Barthes, Roland, Le discours amoureux. Séminaire à l'École pratique des hautes études 19741976, suivi de Fragments d'un discours amoureux, Paris, Seuil, 2007.

—, «Entretien » avec Alain Poirson (1978), in: Barthes, Euvres complètes, nouvelle éd. revue, et prés. par E. Marty, 5 vol., Paris, Seuil, 2002, vol. v, p. 541-544.

—, «Entretien » avec Philippe Roger (1977), in: Barthes, Euvres complètes, op. cit., vol. v, p. 405-417.

—, Fragments d'un discours amoureux (1977), in: Barthes, Euvres complètes, op. cit., vol. v, p. 25-296.

—, «Il luogo comune », Enciclopedia, VIII, Labirinto-Memoria, Turin, Einaudi, 1979.

—, «Leçon » (1978), in : Barthes, Euvres complètes, op. cit., vol. v, p. 429-446.

—, « La linguistique du discours » (1970), in: Barthes, Euvres complètes, op. cit., vol. III, p. 611-616.

—, «Roland Barthes met le langage en question»(1975), in: Barthes, Euvres complètes, op. cit., vol. IV, p. 914-918.

Beck, Ulrich et Elisabeth Beck-Gernsheim, Das ganz normale Chaos der Liebe, Francfort M., Suhrkamp, 1990.

Bruckner, Pascal, Le mariage d'amour a-t-il échoué?, Paris, Grasset, 2010.

De Ruggieri, Francesca, «Frammenti di discorso amoroso fra squilli e SMS : "Il guazzabuglio del linguaggio" », in A. Ponzio, P. Calefato et S. Petrilli (dir.), Con Roland Barthes alle sorgenti del senso, Rome, Meltemi, 2006, p. 499-511.

Ferry, Luc, La révolution de l'amour, Paris, Plon, 2010.

Giddens, Anthony, The Transformation of Intimacy, Stanford, Stanford University Press, 1992.

Illouz, Eva, Consuming the Romantic Utopia. Love and the Cultural Contradictions of Capitalism, Berkeley, University of California Press, 1997. 
Mauss, Marcel, «Les techniques du corps », Journal de psychologie, vol. 32, n 3-4, 1936. Réimp. in Mauss, Sociologie et anthropologie, Paris, P.U.F., coll. Quadrige, 1999, p. 363386. Article disponible à l'adresse Web (consultée le 9 mai 2011): classiques.uqac.ca/classiques/mauss_marcel/socio_et_anthropo/6_Techniques_corps/tech niques_corps.pdf

Ricoeur, Paul, Amour et justice, Paris, Seuil, 2008 ( $1^{\text {ère } e ́ d . ~: ~ T u ̈ b i n g e n, ~ M o h r, ~ 1990) . ~}$

Rorty, Amélie O., «The Historicity of Psychological Attitudes », Midwest Studies in Philosophy, vol. 10, 1986, p. 399-412.

Soncini, Guia, «Gli uomini sono le nuove donne », D-La Repubblica, ${ }^{\circ}$ 722, 4 déc. 2010, p. 79-83.

Watzlawick, Paul, John H. Weakland et Richard Fisch, Change. Principles of Problem Formation and Problem Resolution, New York, W.W. Norton \& Cie, 1974. 